\title{
Philosophiques
}

\section{Émotion et perception}

Étude critique de Tappolet, Christine, Émotions et valeurs, Paris, Presses Universitaires de France, 2000, 296 p.

\section{Paul Dumouchel}

Volume 29, numéro 2, automne 2002

La démocratie délibérative

URI : https://id.erudit.org/iderudit/006261ar

DOI : https://doi.org/10.7202/006261ar

Aller au sommaire du numéro

Éditeur(s)

Société de philosophie du Québec

ISSN

0316-2923 (imprimé)

1492-1391 (numérique)

Découvrir la revue

Citer cet article

Dumouchel, P. (2002). Émotion et perception : étude critique de Tappolet, Christine, Émotions et valeurs, Paris, Presses Universitaires de France, 2000, 296 p. Philosophiques, 29(2), 371-377. https://doi.org/10.7202/006261ar d'utilisation que vous pouvez consulter en ligne.

https://apropos.erudit.org/fr/usagers/politique-dutilisation/ 


\title{
Étude critique
}

\section{Émotion et perception}

Étude critique de Tappolet, Christine, Émotions et valeurs, Paris, Presses Universitaires de France, 2000, 296 p.

\author{
PAUL DUM OUCHEL \\ CRÉA et UQAM \\ Dumouchel.paul@uqam.ca
}

Christine Tappolet avance dans son livre plusieurs hypothèses originales au sujet des émotions, des valeurs et de leur rapport à nos croyances morales. Entre autres, elle reprend une thèse qui avait déjà été proposée par $\mathrm{H}$ usserl, et défendu par $M$ einong, selon laquelle les émotions constituent des perceptions des valeurs. Or cette thèse, semble-t-il, implique le réalisme de valeurs, puisqu'il n'est de perception que de ce qui existe, réalisme que par ailleurs elle revendique. Une des idées les plus novatrices qu'elle défend est que cette perception des valeurs, à l'instar de la perception des couleurs, aurait un contenu non-conceptuel. Cet ensemble structuré d'affirmations s'inscrit en fait au sein d'une démarche d'épistémologie morale, car ce que vise Christine Tappolet en dernier ressort c'est à fonder nos croyances axiologiques et c'est ce à quoi servent ici les émotions. A près avoir montré les limites et les difficultés des approches cohérentistes et fondamentalistes en épistémologie morale, Christine Tappolet avance que les émotions vues comme un outil de perception des valeurs peuvent justifier nos croyances axiologiques.

À la fois pour des raisons de compétence, et parce que je m'accommode assez bien du scepticisme moral, c'est-à-dire de l'idée que nos croyances axiologiques n'ont pas de fondement ultime, dans ce qui va suivre, je ne m'inté resserai pas aux analyses, souvent très détaillées et toujours rigoureuses, de l'épistémologie morale qu'elle présente. II y a là une certaine injustice de la part de l'auteur d'une étude critiquej'en conviens, puisque celles-ci occupent plus de la moitié de l'ouvrage. Je ne m'attarderai dans ce qui suit qu'à la seule thèse qui veut que les émotions constituent des perceptions des valeurs et à certains aspects de celle selon laquelle ces perceptions ont un contenu non-conceptuel. D'une part parce que cette thèse est centrale, c'est sur elle que repose l'ensemble de l'édifice proposé ici, d'autre part parce que dans l'état actuel elle me semble peu convaincante. En effet on peut, mieux, on doit, je crois, reprocher à Christine Tappolet de consacrer trop peu d'espace et de temps à une thèse à la fois aussi importante et difficile, et aussi intéressante. Je trouve d'ailleurs étonnant qu'elle n'accorde qu'un seul chapitre à l'analyse et à la défense de cette idée stimulante et contestable, laquelle constitue selon moi l'aspect le plus original de son ouvrage. 
La thèse repose dans une large mesure sur une analogie entre les émotions, plus particulièrement entre certaines émotions, les émotions occurrentes, et la perception des couleurs (p.168-170 $)^{1}$. De même que la perception est le moyen par lequel nous avons l'expérience des couleurs, les émotions occurrentes seraient celui par lequel nous faisons l'expérience des valeurs. $\mathrm{Ou}$, pour le dire autrement, selon Christine Tappolet, les émotions sont aux valeurs ce que la perception est aux couleurs. Elle évoque trois points pour préciser cette analogie. Premièrement, les émotions tout comme les perceptions sont en général causées par ce qui nous entoure. Deuxièmement, les expériences perceptives et les émotions possèdent toutes deux des propriétés phénomé nales. Troisièmement enfin, ni les unes ni les autres ne sont directement soumises à la volonté. $M$ ais cela ne suffit pas car le lien étroit qui depuis longtemps a été reconnu entre les émotions et les valeurs, suggère encore l'idée d'émotion appropriée. En effet, le rapport qui associe l'émotion à la valeur peut être aberrant ou déviant. On peut se réjouir du malheur d'autrui, s'attrister de son bonheur, envier son succès ou rechercher l'humiliation. $D$ ans tous ces cas, semble-t-il, l'émotion n'est pas adéquate. $N$ e faut-il pas au contraire se réjouir du bonheur, s'attrister du malheur et fuir l'humiliation? Et que faut-il au juste envier?... D'où l'idée que les émotions ne peuvent justifier nos croyances axiologiques que dans la mesure où elles sont appropriées. Car c'est à cette condition seulement qu'une émotion est capable de nous livrer correctement une valeur. D ès lors, propose Christine Tappolet, de même que nos perceptions peuvent être incorrectes et nous amener à croire ce qui est inexact, nos émotions peuvent être inappropriées. Or c'est là me semble-t-il que premièrement le bât blesse. L'exigence qui est en jeu lorsqu'on affirme que la perception possède des conditions de correction est d'un tout autre ordre que celle qui est présente dans l'idée d'émotion appropriée. C'est pourquoi la tentative d'asseoir, ou plutôt d'identifier la seconde de ces notions à la première, me semble vouée à l'échec.

II est vrai que ce n'est pas tout à fait ce que propose Christine Tappolet. Dans la section de son livre consacrée au caractère approprié des émotions, elle analyse trois façons dont les émotions peuvent être évaluées et dites appropriées. Au chapitre précédent elle en avait déjà présenté une autre. Elle ne prétend jamais que ces quatre modes d'évaluation des émotions sont identiques ou dérivent les uns des autres. J e me propose de montrer cependant que seul le premier correspond à peu près aux conditions de correction de la perception des

1. Tous les numéros de page entre parenthèses dans le texte renvoient au livre de Christine Tappolet Emotions et valeurs. On peut par ailleurs se demander qu'elle est la raison de cette restriction aux émotions occurrentes. Les couleurs, en général, ne nous apparaissent pas comme des événements indépendants, mais attachées à des objets, et, comme le remarque le langage courant, la perception d'une scène baignée dans une couleur, le rouge, le vert, le gris, fait souvent penser à la perception d'une situation où domine une tonalité affective particulière. 
couleurs, mais qu'il n'est pas suffisant pour fonder la notion «d'être appropriée» qui lie une émotion et un concept axiologique. Si cette démonstration est exacte, il s'ensuit que la mise en rapport des expériences perceptives et émotives possède peut-être une grande valeur heuristique, mais qu'elle ne révèle pas, contrairement à ce que prétend l'auteur, une analogie suffisamment étroite pour justifier nos croyances axiologiques sur la base de nos « expériences émotives».

A fin de le montrer, il convient premièrement de s'arrêter à la thèse selon laquelle les émotions ont un contenu non-conceptuel. Tappolet reprend de

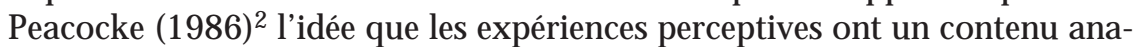
logique plutôt que conceptuel et représente ensuite le contenu non-conceptuel des émotions à la manière de ce contenu analogique. Un contenu est analogique, nous dit-elle, «si, et seulement si, il y a une dimension de variation dans quelque magnitude perceptible telle que pour chaque paire de points sur cette dimension, il peut y avoir une différence correspondante dans le contenu» (p.162). L'idée semble être plus ou moins la suivante, du moins si on pense à la perception des formes ou des couleurs. Le long du spectre lumineux, pour toute variation de l'amplitude, de la longueur d'onde ou même de la pureté de la source lumineuse, nous percevront, ou plutôt nous pouvons percevoir, une couleur ou une teinte différente. L'idée de conditions de correction de nos perceptions est simplement que notre expérience perceptive du rouge est adéquate si et seulement si la longueur d'onde perçue correspond au «rouge». Si, dans ces mêmes circonstances, pour cette même longueur d'onde, nous faisons au contrairel'expérience du «vert» ou du « gris» les conditions de correction ne sont pas satisfaites et on peut dire que l'expérience est trompeuse ${ }^{3}$. Christine Tappolet propose qu'il en va de même des émotions à la différence près qu'en l'occurrence la dimension de variation est celle de l'intensité de la valeur. Pour toute variation de l'intensité de la valeur, il y aura une variation correspondante de l'intensité de l'émotion. Ainsi un danger plus grand commandet-il une peur plus forte, ou du moins doit-on penser qu'il le devrait, et lorsqu'il le fait les conditions de correction de l'émotion sont satisfaites et l'émotion est appropriée.

II existe cependant une différence importante entre la perception des couleurs et l'expérience émotive que Christine Tappolet mentionne à la page 163 mais sans en tirer, me semble-il, toutes les conséquences pour la thèse qu'elle défend. Les variations le long du spectre lumineux engendrent l'expérience des diverses couleurs. Passer du vert au jaune, ce n'est que se déplacer le long de ce spectre. Comme elle le remarque, il en va tout autrement dans le cas des émotions. Si on peut penser que la terreur correspond à une intensité plus élevée de la même val eur que la peur, par exemple le danger, il est plus

2. Peacocke, C., «Analogue Content», Proceedings of the A ristotelian Society, 93, 1986, pp. 203-230.

3. En fait, les choses ne sont pas tout à fait aussi simples comme nous le verrons tout à I'heure lorsqu'il sera question de la constance des couleurs perçues. 


\section{4 - Philosophiques / Automne 2002}

difficile d'avancer que la beauté, le juste, la générosité, le dangereux, l'intéressant, la lâcheté et la cruauté constituent des variations d'intensité d'une même «magnitude». En fait, ce qui est affirmé au premier chapitre, que les valeurs sont incommensurables (p.18), ou qu'elles pourraient au mieux être ordonnées selon une hiérarchie «lexicographique» ${ }^{4}(p .19)$ suggère que l'espace des valeurs ne possède aucune métrique et de toute façon exclu que le passage de l'une à l'autre puisse être représenté par quelque chose d'aussi simple que la croissance linéaire d'un seul paramètre. Dans ces conditions, la comparaison du contenu analogique de la perception avec le contenu nonconceptuel des émotions pourrait rendre compte de ce que l'intensité d'une émotion est appropriée à l'intensité d'une valeur, mais pas du fait qu'une émotion est appropriée à une valeur plutôt qu'une autre. Si dans le cas des couleurs la chose est possible, c'est parce que les différentes couleurs correspondent à des variations du spectre lumineux, mais si les valeurs sont incommensurables c'est alors par définition qu'il n'existe aucune magnitude dont les variations peuvent être corrélées avec les différentes valeurs.

Dans ces conditions, il n'y a, je crois, que deux possibilités si on veut rendre compte du caractère approprié des émotions par rapport aux valeurs. La première, dans la mesure où on veut conserver l'analogie avec la perception visuelle et ses conditions de correction, consiste à rejeter l'idée que les valeurs sont incommensurables et à construire un espace où les différentes valeurs correspondent à des variations de certaines grandeurs mesurables ${ }^{5}$. La seconde consiste à dével opper un concept différent de « être approprié», mais l'analogie avec la perception visuelle en est réduite pour autant. Bien qu'elle ne le dise pas explicitement c'est, je crois, ce que fait en dernier ressort Christine Tappolet. M ais avant de tenter de le montrer, je voudrais revenir au lien entre l'intensité des émotions et l'intensité des valeurs.

A première vue, il semble que pour ce qui est de l'intensité des valeurs au moins les émotions ont bel et bien un contenu analogique. Cependant lorsqu'on y regarde d'un peu plus près la comparaison révèle rapidement ses limites. Q ue l'intensité de l'émotion varie parfois en raison directe de l'intensité de la valeur avec laquelle elle peut être corrélée correspond effectivement à un aspect de notre expérience émotive. $M$ ais l'intensité des émotions varie aussi de manière régulière en raison d'autres facteurs. Ainsi le même malheur

4. L'expression est de moi, Paul Dumouchel. Christine Tappolet reprend une idée de M.N . Lemos (1993) selon laquelle un bien est supérieur à un autre dans la mesure où aucune quantité du second ne pourra être meilleure que la moindre quantité du premier. La parenté avec l'idée d'ordre lexicographique proposée par Rawls (1971) est évidente, d'où l'affirmation que les valeurs rangées selon une telle procédure formerait une hiérarchie lexicographique.

5. Vraisemblablement il s'agira d'un espace complexe à plusieurs dimensions plutôt que le simple déplacement linéaire le long d'une seule dimension comme le suggère l'idée de degré d'intensité. On trouvera un exemple intéressant d'une tentative de ce genre dans le petit livre de Raymond Ruyer Le monde des valeurs (Paris: Aubier, 1948), au premier chapitre intitulé «Valeurs et couleurs». Je remercie Lucien Scubla d'avoir attirer mon attention sur ce texte et pour ses commentaires judicieux sur l'ensemble de cette étude critique. 
me touchera plus s'il frappe mon fils ou une amie proche que s'il s'abat sur un étranger que je ne connais guère. Je réagis aussi généralement plus violemment aux insultes qui me sont faites qu'à celles qui vous font vous plaindre. Celles-là ne semblent-elles pas presque toujours beaucoup plus légères? 0 n peut certes avancer que ma réaction émotive dans ces cas n'est pas adéquate et que mon émotion n'est pas appropriée. $M$ ais une telle réponse soulève au moins deux difficultés.

La première tient à la régularité et à la fréquence de certains de ces phénomènes. II va ainsi par exemple du fait que nos réactions affectives sont généralement plus fortes lorsque nous sommes ou nos proches sont directement impliqués, même si une valeur identique est en jeu et que nous avons par ailleurs des raisons de croire qu'elle est présente à la même « intensité». 0 r, dans le cas des expériences perceptives si nous repérons un infléchissement régulier de la capacité de notre expérience à suivre les variations lumineuses, nous ne concluons pas que notre expérience visuelle est régulièrement inadé quate, bien au contraire. En fait un tel infléchissement existe. N ous savons en effet notre système nerveux «stabilise» notre perception des couleurs, qu'il impose une certaine constance à la couleur des objets perçus, car s'il ne le faisait pas les objets nous sembleraient changer de couleurs sans arrêt en raison des modifications de l'éclairage. Un tel infléchissement, en parti parce qu'il est régulier plutôt qu'un accident, ne nous semble pas une erreur mais nous apparaît au contraire révélateur d'une des fonctions du système perceptif. Pourquoi dans le cas des émotions et de la «perception» des valeurs sommes-nous, semble-t-il, prêt à affirmer au contraire que tous se trompent ${ }^{6}$ ?

La seconde difficulté est étroitement liée à la première. N otre différence spontanée de réaction face à une déviation régulière dans la perception des couleurs ou dans l'émotion des valeurs suggère que le critère auquel nous avons recours pour dire inappropriée l'augmentation de l'intensité de l'émotion en raison de l'intérêt subjectif de l'agent est d'un autre ordre que celui par lequel on définit les conditions de correction d'une expérience perceptive. Dans le cas de la perception des couleurs «être approprié» décrit une relation naturelle qui associe une variation de l'expérience perceptive à toute variation d'une grandeur perceptible. Dans le cas de l'intensité des émotions «être approprié» fait au contraire déjà appel à un concept axiologique. C'est ce que vient confirmer, je crois, le fait que le jugement de valeur par lequel une émotion est dite appropriée ou inappropriée est hautement contestable. Imaginez une femme qui est une utilitariste convaincue (je suis sûr que cela existe) mise en position de venir en aide à un seul de deux enfants qui sont en danger de mort. L'un est son fils, qui est un peu niais et elle le regrette, l'autre une camarade de classe

6. Raymond Ruyer dans Le monde des valeurs, (op. cit., p. 35-38) avance qu'un phénomène similaire se produit dans la perception des valeurs, mais pour le montrer et $y$ donner un autre sens que celui d'une simple erreur, il faut tenir compte d'autres émotions que les seules émotions occurrentes. 


\section{6 - Philosophiques / Automne 2002}

de celui-ci, véritable petit génie dont l'utilité future à la société, si elle vit, sera sûrement fort grande, alors que son fils sera probablement un poids et au mieux ni utile ni nuisible. Condamnée à choisir, elle sauve la génie et laisse mourir son fils. Si elle l'a fait sur la force de ses émotions qui reflétaient fidèlement l'intensité des valeurs en jeu, la plupart jugeront que ses émotions n'étaient pas appropriées. Si elle l'a fait contre ses émotions nous jugerons que son action était inappropriée, qu'elle aurait dû écouter ses émotions plutôt que respecter ses valeurs. On peut aussi penser qu'elle aurait dû avoir d'autres valeurs. $M$ ais dans tous ces cas pour justifier notre condamnation de son acte ou de ses émotions nous ferons appel au lien subjectif qui devrait I'attacher à son enfant. Car même si elle accorde une valeur égale à toute vie humaine, il nous semble que dans une telle circonstance elle devrait privilégier son enfant. Si elle ne le fait pas, nous croyons qu'elle doit s'en expliquer. Si elle n'en ressent pas le besoin, elle nous semblera étrange, monstrueuse presque, incompréhensible, si du moins nous ne la soupçonnons pas de malveillance à l'égard de son enfant. Or le biais subjectif régulier sur lequel nous appuyons tous ces jugements, peut-il se réduire à la corrélation entre l'intensité d'une expérience et la variation d'une grandeur perceptible, en l'occurrence l'intensité de la valeur? L'idée même de biais n'implique-t-elle pas qu'il s'agit d'un écart par rapport à une norme perçue par ailleurs?

Toutes ces difficultés renvoient à une problématique que Christine Tappolet n'aborde jamais directement mais qui, me semble-t-il, est néanmoins au cœur de sa tentative, celle de la naturalisation. Concevoir I'analogie entre perceptions et émotions de façon suffisamment étroite pour que les expériences émotives puissent justifier les croyances axiologiques de même que nos perceptions justifient nos croyances perceptives exige de naturaliser le concept «d'être appropriée» pour une émotion de même qu'on peut naturaliser les conditions de correction de la perception. Les remarques qui pré cèdent ne visent pas à montrer qu'une telle naturalisation est impossible. Elles suggèrent cependant que même dans le cas qui semble être le plus favorable parmi ceux invoqués par Christine Tappolet, celui du rapport entrel'intensité d'une émotion et l'intensité d'une val eur, la notion «d'être appropriée» pour une émotion est elle-même un concept axiologique. II s'ensuit deux consé quences. Premièrement, on voit mal comment les émotions appropriées peuvent justifier les croyances axiologiques si la notion d'émotion appropriée est elle-même un concept axiologique. Car qu'est-ce qui justifie alors ma croyance axiologique que mon émotion est appropriée, ou du moins qu'elle n'est pas inappropriée? R ien ou bien une autre émotion appropriée. II y aura alors régression infinie. Deuxièmement, il me semble que si on est incapable de rendre compte de façon naturaliste de la notion « d'émotion appropriée», la perception des valeurs risque d'apparaître comme un phénomène étrange et mystérieux, guère plus compréhensible que «l'improbable faculté de perception des valeurs» que selon elle l'intuitionnisme «se voit obligé de postuler» (p. 124). La naturalisation quej'ai en tête n'exige pas qu'on exhibe le 
système matériel responsable de la perception des valeurs ou encore qu'on ancre cette perception dans la biologie, mais simplement qu'on l'explique en ne faisant appel qu'à des catégories des sciences naturelles.

$M$ ais il est aussi une autre raison, plus simple et évidente qui pousse à penser que l'analogie entre émotion et perception, du moins sous la forme où elle nous est présentée ici, atteint rapidement ses limites. Le premier point que Christine Tappolet invoque pour préciser cette analogie est le fait que les « expériences perceptuelles et les émotions sont en général causées par les choses qui nous environnent. » (p.168) Ceci est vrai, mais cette affirmation cache, je crois, une dis-analogie beaucoup plus importante. En effet, les perceptions sont toujours trompeuses lorsqu'elles ne sont pas causées par les choses qui nous entourent. II n'y a pas de perception (véridique) de ce qui est passé, absent ou fictif. II n'y a là que rêve, illusion, illumination ou une imagination trop enflammée. La raison en est que la perception définit pour nous ce qui est présent. $\mathrm{O} r$ il en va tout autrement des émotions. $\mathrm{N}$ ul ne pensera que ma tristesse et mon horreur en apprenant plusieurs années plus tard les circonstances dramatiques de la mort d'un de mes amis, sont inappropriées ou illusoires. O n peut certes répondre que les valeurs que mes émotions «perçoivent » alors sont présentes dans le récit. $M$ ais dans ce cas, tout comme dans celui où l'émotion donne accès à des valeurs qui sont absentes dans l'espace et le temps, ne faut-il pas avouer que la « perception émotive» ressemble plus à la compréhension intellectuelle qu'à l'expérience visuelle?

Il faut avoir gré je crois à Christine Tappolet d'avoir défendu avec rigueur et des outils analytiques puissants une thèse difficile et provocante et d'avoir mis en évidence par la même occasion certaines des contradictions et des difficultés qu'on trouve à l'heure actuelle en épistémologie morale. La thèse centrale au sujet des émotions qu'elle défend, et sur laquelle repose sa proposition de justification de nos énoncés axiologiques, présente cependant dans l'état actuel encore trop de problèmes pour être, selon moi, entièrement convaincante. 